Article

\title{
On the Fractional Maximal Delta Integral Type Inequalities on Time Scales
}

\section{Lütfi Akın}

Department of Bussiness Administration, Mardin Artuklu University, Mardin 47100, Turkey; lutfiakin@artuklu.edu.tr

Received: 8 May 2020; Accepted: 15 June 2020; Published: 17 June 2020

\begin{abstract}
Time scales have been the target of work of many mathematicians for more than a quarter century. Some of these studies are of inequalities and dynamic integrals. Inequalities and fractional maximal integrals have an important place in these studies. For example, inequalities and integrals contributed to the solution of many problems in various branches of science. In this paper, we will use fractional maximal integrals to establish integral inequalities on time scales. Moreover, our findings show that inequality is valid for discrete and continuous conditions.
\end{abstract}

Keywords: operator theory; time scales; integral inequalities

MSC: 47B38; 34N05; 35A23

\section{Introduction}

The founder of the study of dynamic equations on time scales is Stefan Hilger [1]. Recently, the inequalities and dynamic equations on time scales have received great attention. Dynamic equations and inequalities have many applications in quantum mechanics, neural networks, heat transfer, electrical engineering, optics, economy and population dynamics [2-5]. It is possible to give an example from the economy, seasonal investments and income [6]. Many mathematicians have demonstrated various aspects of integral inequalities on time scales $[7,8]$. The most important examples of time scale studies are differential, difference and quantum calculus [9], i.e., when $\mathbb{T}=\mathbb{R}, \mathbb{T}=\mathbb{N}$ and $\mathbb{T}=q^{\mathbb{N}_{0}}=\left\{q^{t}: t \in \mathbb{N}_{0}\right\}$ where $q>1$.

Fractional calculus is an extended version of non-integer integrals and derivatives. In time scales, Lebesgue spaces and different spaces, the subject of fractional integrals has been studied by many mathematicians [10-19]. We consider the functional [20]:

$$
T(f, g)=\frac{1}{t-a} \int_{a}^{t} f(x) g(x) \Delta x-\left(\frac{1}{t-a} \int_{a}^{t} f(x) \Delta x\right)\left(\frac{1}{t-a} \int_{a}^{t} g(x) \Delta x\right)
$$

where $f$ and $g$ are two synchronous integrable functions on $[a, t]_{\mathbb{T}}$, (i.e., $[a, t]_{\mathbb{T}}=[a, t] \cap \mathbb{T}$ ).

The main subject of our article is to create new fractional inequalities by using fractional maximal integral operators and synchronous functions on time scales. In addition, our findings include continuous inequalities and corresponding discrete analogs.

The organization of this article is as follows. In Section 2, we will give some definitions of the $\Delta$-maximal type fractional integral operator on time scales. In Section 3, we will create new fractional inequalities by using fractional maximal integral operator and synchronous functions on time scales. In Section 4, we show a few applications of our results. 


\section{Preliminaries}

Some basic concepts related to time scale are given below without proof. We recommend that the reader refer to the [2-25] monographs for details.

Definition 1. [21] Given an open set $\Omega \subset \mathbb{R}^{n}$ and $a, 0<a<n$. Fractional maximal operator $M_{a}$ f is defined as follows

$$
\mathrm{M}_{\mathrm{a}} \mathrm{f}(\mathrm{t})=\sup _{\mathrm{B} \ni \mathrm{t}} \frac{1}{|\mathrm{~B}|^{1-\frac{\mathrm{a}}{\mathrm{n}}}} \int_{\mathrm{B} \cap \Omega} \mathrm{f}(\mathrm{y}) \mathrm{dy}
$$

where the supremum is again taken over all balls $B$ which contain $t$. In the limiting case $a=0$, the fractional maximal operator reduces to the Hardy-Littlewood maximal operator.

Definition 2. [21] Let $\Phi \subset \mathbb{R}$ and $p: \Phi \rightarrow[1, \infty)$ a measurable function. $L^{p(.)}$ is composed of all measurable functions $f$ on $\Phi$ such that

$$
\int_{\Phi}\left(\frac{|\mathrm{f}(\mathrm{x})|}{\lambda}\right)^{\mathrm{p}(\mathrm{x})} \mathrm{dx} \leq 1
$$

for any $\lambda>0$. The norm in $L^{p(x)}$ space is the generalization of the norm in $L^{p}$ space (p is constant). The Luxemburg norm in $L^{p(x)}$ space is defined as follows.

$$
\|f\|_{L^{p(.)}}=\inf \left\{\lambda>0: \int_{\Phi}\left(\frac{|f(x)|}{\lambda}\right)^{p(x)} d x \leq 1\right\}
$$

At the same time $L^{p(x)}$ becomes a Banach space.

Definition 3. [22] Let $f$ and $g$ be two integrable functions on $[a, t]_{\mathbb{T}}\left(\right.$ i.e., $\left.[a, t]_{\mathbb{T}}=[a, t] \cap \mathbb{T}\right)$. If for any $x, y \in[a, t]_{\mathbb{T}}$

$$
[\mathrm{f}(\mathrm{x})-\mathrm{f}(\mathrm{y})][\mathrm{g}(\mathrm{x})-\mathrm{g}(\mathrm{y})] \geq 0,
$$

then $f$ and $g$ are called synchronous real-valued functions on $[a, t]_{\mathbb{T}} \subset \mathbb{R}$.

Definition 4. [23] A time scale $\mathbb{T}$ is an arbitrary nonempty closed subset of the real numbers $\mathbb{R}$. We define the forward jump operator $\sigma: \mathbb{T} \rightarrow \mathbb{T}$ by $\sigma(t)=\inf \{s \in \mathbb{T}: s>t\}$ for $t \in \mathbb{T}$ and we define the backward jump operator $\rho: \mathbb{T} \rightarrow \mathbb{T}$ as defined by $\rho(t)=\sup \{s \in \mathbb{T}: s<t\}$ for $t \in \mathbb{T}$.

If $\sigma(t)>t$, we say that $t$ is right-scattered and if $\rho(t)<t$, we say that $t$ is left-scattered. Moreover, if $\sigma(t)=t$, then $t$ is called right-dense and if $\rho(t)=t$, then $t$ is called left-dense. $\mu: \mathbb{T} \rightarrow \mathbb{R}^{+}$such that $\mu(t)=\sigma(t)-t$ is called graininess mapping.

If $\mathbb{T}$ has a left-scattered maximum $m$, then $\mathbb{T}^{k}=\mathbb{T}-\{m\}$. Otherwise $\mathbb{T}^{k}=\mathbb{T}$. Briefly

$$
\mathbb{T}^{\mathrm{k}}=\left\{\begin{array}{r}
\mathbb{T} \backslash(\operatorname{\rho sup} \mathbb{T}, \sup \mathbb{T}], \quad \text { if } \quad \sup \mathbb{T}<\infty, \\
\mathbb{T}, \quad \text { if } \quad \sup \mathbb{T}=\infty .
\end{array}\right.
$$

Along the same lines

$$
\mathbb{T}_{k}=\left\{\begin{array}{rr}
\mathbb{T} \backslash[\inf \mathbb{T}, \sigma(\inf \mathbb{T})], & |\inf \mathbb{T}|<\infty, \\
\mathbb{T}, & \inf \mathbb{T}=-\infty
\end{array}\right.
$$

Definition 5 [23] A function $f:[a, b] \rightarrow \mathbb{R}$ is said to be right-dense continuous if it is right continuous at each right-dense point and there exists a finite left limit at all left-dense points, and $f$ is said to be differentiable if its derivative exists.

The space of $r d$-continuous functions is denoted by $C_{r d}(\mathbb{T}, \mathbb{R})$. 
Definition 6. [23] The generalized polynomials, that also occur in Taylor's formula are $g_{k}, h_{k}: \mathbb{T}^{2} \rightarrow \mathbb{R}, k \in \mathbb{N}_{0}$ functions. The functions $g_{0}, h_{0}$ are $g_{0}(t, s)=h_{0}(t, s) \equiv 1, \forall s, t \in \mathbb{T}$. Given $g_{k+1}$ and $h_{k+1}$ are

$$
\mathrm{g}_{\mathrm{k}+1}(\mathrm{t}, \mathrm{s})=\int_{\mathrm{s}}^{\mathrm{t}} \mathrm{g}_{\mathrm{k}}(\sigma(\tau), \mathrm{s}) \Delta \tau, \forall \mathrm{s}, \mathrm{t} \in \mathbb{T}
$$

and

$$
h_{k+1}(t, s)=\int_{s}^{t} h_{k}(\tau, s) \Delta \tau, \forall s, t \in \mathbb{T}
$$

We claim that for $k \in \mathbb{N}_{0}$

$$
\mathrm{g}_{\mathrm{k}}(\mathrm{t}, \mathrm{s})=\mathrm{h}_{\mathrm{k}}(\mathrm{t}, \mathrm{s})=\frac{(\mathrm{t}-\mathrm{s})^{\mathrm{k}}}{\mathrm{k} !} \forall \mathrm{s}, \mathrm{t} \in \mathbb{R}
$$

Definition 7. [24] If $f \in C_{r d}(\mathbb{T}, \mathbb{R})$ and $t \in \mathbb{T}^{k}$, then

$$
\int_{t}^{\sigma(t)} f(\tau) \Delta \tau=\mu(t) f(t)
$$

For $\alpha \geq 1$ we can define the time scale $\Delta$-maximal type fractional integral

$$
\begin{gathered}
M_{a}^{\alpha} f(t)=\sup _{B \ni t} \frac{1}{|B|^{\frac{n-a}{n}}} \int_{a}^{t} h_{\alpha-1}(t, \sigma(\tau)) f(\tau) \Delta \tau, \\
M_{a}^{0} f(t)=\sup _{B \ni t} \frac{1}{|B|^{1-\frac{a}{n}}} f(t),
\end{gathered}
$$

where $f \in L_{1}([a, t] \cap \mathbb{T})$ and $M_{a}^{\alpha} f \in L_{1}([a, t] \cap \mathbb{T})$ (for details on Lemma 2, see [25]) Lebesgue $\Delta$-integrable functions on $[a, t] \cap \mathbb{T}, t \in[a, t] \cap \mathbb{T}$.

Lemma 1. (Lemma 2, [25]) Let $\alpha \geq 1, f \in L_{1}([a, t] \cap \mathbb{T})$. Assume $h_{\alpha-1}(t, \sigma(\tau))$ is additionally Lebesgue $\Delta$-measurable on $([a, t] \cap \mathbb{T})^{2} ; a, t \in \mathbb{T}$. Then $M_{a}^{\alpha} f \in L_{1}([a, t] \cap \mathbb{T})$.

\section{Main Result}

We now present the inequalities with respect to fractional maximal integral type operators and their norms in the variable exponential Lebesgue space.

Theorem 1. Let $f$ and $g$ be two real-valued synchronous functions on $[0, \infty)_{\mathbb{T}} \subset \mathbb{R}$. For $\forall t>a, a>0, \alpha \geq 1$ we have

$$
M_{a}^{\alpha}(f g)(t) \geq\left(h_{\alpha}(t, a)\right)^{-1}\left(M_{a}^{\alpha} f\right)(t)\left(M_{a}^{\alpha} g\right)(t) .
$$

Proof. If $\mathrm{f}$ and $\mathrm{g}$ are two synchronous functions on $[0, \infty)_{\mathbb{T}}$, then, for $\forall \tau, \theta \geq 0$,

$$
f(\tau) g(\tau)-f(\tau) g(\theta)-f(\theta) g(\tau)+f(\theta) g(\theta) \geq 0
$$

Hence

$$
f(\tau) g(\tau)+f(\theta) g(\theta) \geq f(\tau) g(\theta)+f(\theta) g(\tau)
$$

For $\tau \in(a, t)$ multiplying both sides of (2) by $h_{\alpha-1}(t, \sigma(\tau))$ we have

$$
\begin{aligned}
& h_{\alpha-1}(t, \sigma(\tau)) f(\tau) g(\tau)+h_{\alpha-1}(t, \sigma(\tau)) f(\theta) g(\theta) \geq h_{\alpha-1}(t, \sigma(\tau)) f(\tau) g(\theta)+ \\
& h_{\alpha-1}(t, \sigma(\tau)) f(\theta) g(\tau) .
\end{aligned}
$$


If we take the integral of both sides of (3) through $(a, t)$ we get

$$
\begin{aligned}
& \int_{a}^{t} h_{\alpha-1}(t, \sigma(\tau)) f(\tau) g(\tau) \Delta \tau+\int_{a}^{t} h_{\alpha-1}(t, \sigma(\tau)) f(\theta) g(\theta) \Delta \tau \\
\geq & \int_{a}^{t} h_{\alpha-1}(t, \sigma(\tau)) f(\tau) g(\theta) \Delta \tau+\int_{a}^{t} h_{\alpha-1}(t, \sigma(\tau)) f(\theta) g(\tau) \Delta \tau .
\end{aligned}
$$

Since $f(\theta), g(\theta)$ and $(f(\theta) g(\theta))$ are independent from $\tau$, we can take them out of integral. Thus, the following is obtained

$$
\begin{gathered}
\int_{a}^{t} h_{\alpha-1}(t, \sigma(\tau)) f(\tau) g(\tau) \Delta \tau+f(\theta) g(\theta) \int_{a}^{t} h_{\alpha-1}(t, \sigma(\tau)) \Delta \tau \\
\geq g(\theta) \int_{a}^{t} h_{\alpha-1}(t, \sigma(\tau)) f(\tau) \Delta \tau+f(\theta) \int_{a}^{t} h_{\alpha-1}(t, \sigma(\tau)) g(\tau) \Delta \tau .
\end{gathered}
$$

If we take $\alpha+1$ instead of $\mathrm{k}\left(\mathrm{h}_{\mathrm{k}+1}(\mathrm{t}, \mathrm{s})=\int_{\mathrm{s}}^{\mathrm{t}} \mathrm{h}_{\mathrm{k}}(\tau, \mathrm{s}) \Delta \tau, \forall \mathrm{s}, \mathrm{t} \in \mathbb{T}\right.$ and $\mathrm{g}_{\mathrm{k}+1}(\mathrm{t}, \mathrm{s})=$ $\left.\int_{s}^{t} g_{k}(\sigma(\tau), s) \Delta \tau, \forall s, t \in \mathbb{T}\right)$ in Definition 6, we get the following.

$$
h_{\alpha}(t, s)=\int_{s}^{t} h_{\alpha-1}(\tau, s) \Delta \tau, \forall s, t \in \mathbb{T}
$$

Similarly,

$$
\mathrm{g}_{\alpha}(\mathrm{t}, \mathrm{s})=\int_{\mathrm{s}}^{\mathrm{t}} \mathrm{g}_{\alpha-1}(\sigma(\tau), \mathrm{s}) \Delta \tau, \forall \mathrm{s}, \mathrm{t} \in \mathbb{T}
$$

We know that,

$$
\sup _{B \ni t} \frac{1}{|B|^{\frac{n-a}{n}}} \int_{a}^{t} h_{\alpha-1}(t, \sigma(\tau)) \Delta \tau \geq \int_{a}^{t} h_{\alpha-1}(t, \sigma(\tau)) \Delta \tau=h_{\alpha}(t, a), \text { for }, 0<a<n .
$$

If we take the supremum of both sides of (4) over B $\ni t$, we get the following

$$
\begin{aligned}
& \sup _{B \ni t} \frac{1}{|B|^{\frac{n-a}{n}}} \int_{a}^{t} h_{\alpha-1}(t, \sigma(\tau)) f(\tau) g(\tau) \Delta \tau+f(\theta) g(\theta) \sup _{B \ni t} \frac{1}{|B|^{\frac{n-a}{n}}} \int_{a}^{t} h_{\alpha-1}(t, \sigma(\tau)) \Delta \tau \\
& \geq g(\theta) \sup _{B \ni t} \frac{1}{|B|^{\frac{n-a}{n}}} \int_{a}^{t} h_{\alpha-1}(t, \sigma(\tau)) f(\tau) \Delta \tau+f(\theta) \sup _{B \ni t} \frac{1}{|B|^{\frac{n-a}{n}}} \int_{a}^{t} h_{\alpha-1}(t, \sigma(\tau)) g(\tau) \Delta \tau
\end{aligned}
$$

Due to $\left(M_{a}^{\alpha} f(t)=\sup _{B \ni t} \frac{1}{|B|^{\frac{n-a}{n}}} \int_{a}^{t} h_{\alpha-1}(t, \sigma(\tau)) f(\tau) \Delta \tau\right)$ Definition 7, we get following

$$
M_{a}^{\alpha}(f g)(t)+f(\theta) g(\theta)\left(h_{\alpha}(t, a)\right) \geq g(\theta)\left(M_{a}^{\alpha} f\right)(t)+f(\theta)\left(M_{a}^{\alpha} g\right)(t) .
$$

For $\theta \in(\mathrm{a}, \mathrm{t})$ multiplying both sides of (5) by $\mathrm{h}_{\alpha-1}(\mathrm{t}, \sigma(\theta))$ we have

$$
\begin{aligned}
& h_{\alpha-1}(t, \sigma(\theta)) M_{a}^{\alpha}(f g)(t)+h_{\alpha-1}(t, \sigma(\theta)) f(\theta) g(\theta)\left(h_{\alpha}(t, a)\right) \\
& \geq h_{\alpha-1}(t, \sigma(\theta)) g(\theta)\left(M_{a}^{\alpha} f\right)(t)+h_{\alpha-1}(t, \sigma(\theta)) f(\theta)\left(M_{a}^{\alpha} g\right)(t) .
\end{aligned}
$$

If we take the integral of both sides of (6) through $(a, t)$ we get

$$
\int_{a}^{t} h_{\alpha-1}(t, \sigma(\theta)) M_{a}^{\alpha}(f g)(t) \Delta \theta+\int_{a}^{t} h_{\alpha-1}(t, \sigma(\theta)) f(\theta) g(\theta)\left(h_{\alpha}(t, a)\right) \Delta \theta
$$




$$
\geq \int_{\mathrm{a}}^{\mathrm{t}} \mathrm{h}_{\alpha-1}(\mathrm{t}, \sigma(\theta)) \mathrm{g}(\theta)\left(\mathrm{M}_{\mathrm{a}}^{\alpha} \mathrm{f}\right)(\mathrm{t}) \Delta \theta+\int_{\mathrm{a}}^{\mathrm{t}} \mathrm{h}_{\alpha-1}(\mathrm{t}, \sigma(\theta)) \mathrm{f}(\theta)\left(\mathrm{M}_{\mathrm{a}}^{\alpha} \mathrm{g}\right)(\mathrm{t}) \Delta \theta
$$

Since $M_{a}^{\alpha}(f g)(t),\left(M_{a}^{\alpha} f\right)(t),\left(M_{a}^{\alpha} g\right)(t)$ and $\left(h_{\alpha}(t, a)\right)$ are independent from $\theta$, we can take them out of integral.

If we take $\alpha+1$ instead of $k$ and if we take $\theta$ instead of $\tau\left(h_{k+1}(t, s)=\int_{s}^{t} h_{k}(\tau, s) \Delta \tau, \forall s, t \in \mathbb{T}\right.$ and $g_{k+1}(t, s)=\int_{s}^{t} g_{k}(\sigma(\tau), s) \Delta \tau, \forall s, t \in \mathbb{T}$ in the Definition 6), we get the following

$$
\int_{\mathrm{a}}^{\mathrm{t}} \mathrm{h}_{\alpha-1}(\mathrm{t}, \sigma(\theta)) \Delta \theta=\mathrm{h}_{\alpha}(\mathrm{t}, \mathrm{a}) .
$$

Thus, the following is obtained

$$
\begin{aligned}
& M_{a}^{\alpha}(f g)(t) \int_{a}^{t} h_{\alpha-1}(t, \sigma(\theta)) \Delta \theta+\left(h_{\alpha}(t, a)\right) \int_{a}^{t} h_{\alpha-1}(t, \sigma(\theta)) f(\theta) g(\theta) \Delta \theta \\
& \geq\left(M_{a}^{\alpha} f\right)(t) \int_{a}^{t} h_{\alpha-1}(t, \sigma(\theta)) g(\theta) \Delta \theta+\left(M_{a}^{\alpha} g\right)(t) \int_{a}^{t} h_{\alpha-1}(t, \sigma(\theta)) f(\theta) \Delta \theta .
\end{aligned}
$$

Hence from Definitions 5 and 6 the following is obtained

$$
M_{a}^{\alpha}(f g)(t)\left(h_{\alpha}(t, a)\right)+\left(h_{\alpha}(t, a)\right) M_{a}^{\alpha}(f g)(t) \geq\left(M_{a}^{\alpha} f\right)(t)\left(M_{a}^{\alpha} g\right)(t)+\left(M_{a}^{\alpha} g\right)(t)\left(M_{a}^{\alpha} f\right)(t) .
$$

Finally we get

$$
M_{a}^{\alpha}(f g)(t)\left(h_{\alpha}(t, a)\right) \geq\left(M_{a}^{\alpha} f\right)(t)\left(M_{a}^{\alpha} g\right)(t)
$$

Theorem 2. Let $f$ and $g$ be two real-valued synchronous functions on $[0, \infty)_{\mathbb{T}} \subset \mathbb{R}$. For $\forall t>a, a>0, \alpha, \beta \geq 1$ we have

$$
h_{\alpha}(t, a) M_{a}^{\beta}(f g)(t)+h_{\beta}(t, a) M_{a}^{\alpha}(f g)(t) \geq\left(M_{a}^{\alpha} f\right)(t)\left(M_{a}^{\beta} g\right)(t)+\left(M_{a}^{\beta} f\right)(t)\left(M_{a}^{\alpha} g\right)(t) .
$$

Proof. If $\mathrm{f}$ and $\mathrm{g}$ are two synchronous functions on $[0, \infty)_{\mathbb{T}}$, then for $\forall \tau, \theta \geq 0$ we have

$$
(f(\tau)-f(\theta))(g(\tau)-g(\theta)) \geq 0 .
$$

Hence

$$
f(\tau) g(\tau)+f(\theta) g(\theta) \geq f(\tau) g(\theta)+f(\theta) g(\tau) .
$$

For $\tau \in(a, t)$ multiplying both sides of $(7)$ by $h_{\alpha-1}(t, \sigma(\tau))$ we have

$$
\mathrm{h}_{\alpha-1}(\mathrm{t}, \sigma(\tau)) \mathrm{f}(\tau) \mathrm{g}(\tau)+\mathrm{h}_{\alpha-1}(\mathrm{t}, \sigma(\tau)) \mathrm{f}(\theta) \mathrm{g}(\theta) \geq \mathrm{h}_{\alpha-1}(\mathrm{t}, \sigma(\tau)) \mathrm{f}(\tau) \mathrm{g}(\theta)+\mathrm{h}_{\alpha-1}(\mathrm{t}, \sigma(\tau)) \mathrm{f}(\theta) \mathrm{g}(\tau)
$$

If we take the integral of both sides of $(8)$ through $(a, t)$ we get

$$
\begin{aligned}
& \int_{a}^{t} h_{\alpha-1}(t, \sigma(\tau)) f(\tau) g(\tau) \Delta \tau+\int_{a}^{t} h_{\alpha-1}(t, \sigma(\tau)) f(\theta) g(\theta) \Delta \tau \\
\geq & \int_{a}^{t} h_{\alpha-1}(t, \sigma(\tau)) f(\tau) g(\theta) \Delta \tau+\int_{a}^{t} h_{\alpha-1}(t, \sigma(\tau)) f(\theta) g(\tau) \Delta \tau .
\end{aligned}
$$

We know that, $\sup _{B \ni t} \frac{1}{|B|^{\frac{n-a}{n}}} \int_{a}^{t} h_{\alpha-1}(t, \sigma(\tau)) \Delta \tau \geq \int_{a}^{t} h_{\alpha-1}(t, \sigma(\tau)) \Delta \tau=h_{\alpha}(t, a)$, for $0<\mathrm{a}<\mathrm{n}$. 
If we take the supremum of both sides of (9) over B $\ni t$ we get the following

$$
\begin{aligned}
& \sup _{B \ni t} \frac{1}{|B|^{\frac{n-a}{n}}} \int_{a}^{t} h_{\alpha-1}(t, \sigma(\tau)) f(\tau) g(\tau) \Delta \tau+\sup _{B \ni t} \frac{1}{|B|^{\frac{n-a}{n}}} \int_{a}^{t} h_{\alpha-1}(t, \sigma(\tau)) f(\theta) g(\theta) \Delta \tau \\
& \geq \sup _{B \ni t} \frac{1}{|B|^{\frac{n-a}{n}}} \int_{a}^{t} h_{\alpha-1}(t, \sigma(\tau)) f(\tau) g(\theta) \Delta \tau+\sup _{B \ni t} \frac{1}{|B|^{\frac{n-a}{n}}} \int_{a}^{t} h_{\alpha-1}(t, \sigma(\tau)) f(\theta) g(\tau) \Delta \tau .
\end{aligned}
$$

Due to $\left(M_{a}^{\alpha} f(t)=\sup _{B \ni t} \frac{1}{|B|^{\frac{n-a}{n}}} \int_{a}^{t} h_{\alpha-1}(t, \sigma(\tau)) f(\tau) \Delta \tau\right)$ Definition 7, we get following

$$
M_{a}^{\alpha}(f g)(t)+h_{\alpha}(t, \sigma(\tau)) f(\theta) g(\theta) \geq g(\theta)\left(M_{a}^{\alpha} f\right)(t)+f(\theta)\left(M_{a}^{\alpha} g\right)(t) .
$$

For $\theta \in(a, t)$ multiplying both sides of $(10)$ by $h_{\beta-1}(t, \sigma(\theta))$ we have

$$
\begin{aligned}
& \mathrm{h}_{\beta-1}(\mathrm{t}, \sigma(\theta)) \mathrm{M}_{\mathrm{a}}^{\alpha}(\mathrm{fg})(\mathrm{t})+\mathrm{h}_{\beta-1}(\mathrm{t}, \sigma(\theta)) \mathrm{h}_{\alpha}(\mathrm{t}, \sigma(\tau)) \mathrm{f}(\theta) \mathrm{g}(\theta) \\
& \geq \mathrm{h}_{\beta-1}(\mathrm{t}, \sigma(\theta)) \mathrm{g}(\theta)\left(\mathrm{M}_{\mathrm{a}}^{\alpha} \mathrm{f}\right)(\mathrm{t})+\mathrm{h}_{\beta-1}(\mathrm{t}, \sigma(\theta)) \mathrm{f}(\theta)\left(\mathrm{M}_{\mathrm{a}}^{\alpha} \mathrm{g}\right)(\mathrm{t}) .
\end{aligned}
$$

If we take the integral of both sides of (11) through $(a, t)$ we get

$$
\begin{aligned}
& \int_{a}^{t} h_{\beta-1}(t, \sigma(\theta)) M_{a}^{\alpha}(f g)(t) \Delta \theta+\int_{a}^{t} h_{\beta-1}(t, \sigma(\theta)) h_{\alpha}(t, \sigma(\tau)) f(\theta) g(\theta) \Delta \theta \\
& \geq \int_{a}^{t} h_{\beta-1}(t, \sigma(\theta)) g(\theta)\left(M_{a}^{\alpha} f\right)(t) \Delta \theta+\int_{a}^{t} h_{\beta-1}(t, \sigma(\theta)) f(\theta)\left(M_{a}^{\alpha} g\right)(t) \Delta \theta .
\end{aligned}
$$

Hereby

$$
\begin{aligned}
& M_{a}^{\alpha}(f g)(t) \int_{a}^{t} h_{\beta-1}(t, \sigma(\theta)) \Delta \theta+h_{\alpha}(t, \sigma(\tau)) \int_{a}^{t} h_{\beta-1}(t, \sigma(\theta)) f(\theta) g(\theta) \Delta \theta \\
& \geq\left(M_{a}^{\alpha} f\right)(t) \int_{a}^{t} h_{\beta-1}(t, \sigma(\theta)) g(\theta) \Delta \theta+\left(M_{a}^{\alpha} g\right)(t) \int_{a}^{t} h_{\beta-1}(t, \sigma(\theta)) f(\theta) \Delta \theta .
\end{aligned}
$$

We get the following result from the above inequality

$$
\begin{gathered}
h_{\alpha}(t, a) M_{a}^{\beta}(f g)(t)+h_{\beta}(t, a) M_{a}^{\alpha}(f g)(t) \\
\geq\left(M_{a}^{\alpha} f\right)(t)\left(M_{a}^{\beta} g\right)(t)+\left(M_{a}^{\beta} f\right)(t)\left(M_{a}^{\alpha} g\right)(t) .
\end{gathered}
$$

Thus, the proof of Theorem 2 is completed.

Theorem 3. Let $\left(f_{i}\right)_{i=1, \ldots, n}$ be $n$ positive increasing functions on $[0, \infty)_{\mathbb{T}}$. For $\forall t>a, a \geq 0, \alpha \geq 1$ we have

$$
M_{a}^{\alpha}\left(\prod_{i=1}^{n} f_{i}\right)(t) \geq\left(h_{\alpha}(t, a)\right)^{1-n} \prod_{i=1}^{n} M_{a}^{\alpha} f_{i}(t) .
$$

Proof. The induction method will be used to prove our theorem. For $n=1$, and $\forall t>a, a \geq 0, \alpha \geq 1$ we have

$$
M_{a}^{\alpha}\left(f_{1}\right)(t) \geq M_{a}^{\alpha} f_{1}(t) .
$$

For $\mathrm{n}=2$ and $\forall \mathrm{t}>\mathrm{a}, \mathrm{a} \geq 0, \alpha \geq 1$ applying Theorem 1 we have

$$
M_{a}^{\alpha}\left(f_{1} f_{2}\right)(t) \geq\left(h_{\alpha}(t, a)\right)^{-1} M_{a}^{\alpha} f_{1}(t) M_{a}^{\alpha} f_{2}(t) .
$$


For $\mathrm{n}=\mathrm{n}-1$ we assume that the following inequality holds.

$$
M_{a}^{\alpha}\left(\prod_{i=1}^{n-1} f_{i}\right)(t) \geq\left(h_{\alpha}(t, a)\right)^{2-n} \prod_{i=1}^{n-1} M_{a}^{\alpha} f_{i}(t) .
$$

For $\mathrm{n}$ we have to prove the following inequality

$$
M_{a}^{\alpha}\left(\prod_{i=1}^{n} f_{i}\right)(t) \geq\left(h_{\alpha}(t, a)\right)^{1-n} \prod_{i=1}^{n} M_{a}^{\alpha} f_{i}(t) .
$$

We know that $\left(f_{i}\right)_{i=1, \ldots, n}$ is a positive increasing function. Thus, $\left(\prod_{i=1}^{n-1} f_{i}\right)(t)$ is a positive increasing function.

Let $\prod_{i=1}^{n-1} f_{i}=f, f_{n}=g$ and applying Theorem 1 we have

$$
\prod_{i=1}^{n} f_{i}=\prod_{i=1}^{n-1} f_{i} f_{n}=f g
$$

and

$$
M_{a}^{\alpha}\left(\prod_{i=1}^{n} f_{i}\right)(t)=M_{a}^{\alpha}\left(\prod_{i=1}^{n-1} f_{i} f_{n}\right)(t) \geq\left(h_{\alpha}(t, a)\right)^{-1} M_{a}^{\alpha}\left(\prod_{i=1}^{n-1} f_{i}\right)(t) M_{a}^{\alpha}\left(f_{n}\right)(t) .
$$

Multiplying both sides of (12) by $\left(h_{\alpha}(t, a)\right)^{-1} M_{a}^{\alpha}\left(f_{n}\right)(t)$ we have

$$
\left(h_{\alpha}(t, a)\right)^{-1} M_{a}^{\alpha}\left(f_{n}\right)(t) M_{a}^{\alpha}\left(\prod_{i=1}^{n-1} f_{i}\right)(t) \geq\left(h_{\alpha}(t, a)\right)^{-1} M_{a}^{\alpha}\left(f_{n}\right)(t)\left(h_{\alpha}(t, a)\right)^{2-n} \prod_{i=1}^{n-1} M_{a}^{\alpha} f_{i}(t) .
$$

Herewith, we get the following result from the above inequality

$$
M_{a}^{\alpha}\left(\prod_{i=1}^{n} f_{i}\right)(t) \geq\left(h_{\alpha}(t, a)\right)^{1-n} \prod_{i=1}^{n} M_{a}^{\alpha} f_{i}(t) .
$$

Theorem 4. Let $\alpha>2, f \in C_{r d}(\mathbb{T})$. Suppose $h_{\alpha-2}(t, \sigma(t))$ to be continuous on $\left([0, \infty)_{\mathbb{T}}\right)^{2}$ with $p, q>$ $1, \frac{1}{p}+\frac{1}{q}=1$. Then we have

$$
\int_{0}^{\infty}\left|\mathrm{M}_{0}^{\alpha} \mathrm{f}(\mathrm{t})\right|^{\mathrm{q}} \Delta \mathrm{t} \leq\left(\int_{0}^{\infty} \sup _{\mathrm{B} \ni \mathrm{t}} \frac{1}{|\mathrm{~B}|}\left(\int_{0}^{\mathrm{t}}\left|\mathrm{h}_{\alpha-2}(\mathrm{t}, \sigma(\tau))\right|^{\mathrm{p}} \Delta \tau\right)^{\frac{\mathrm{q}}{\mathrm{p}}} \Delta \mathrm{t}\right)\left(\int_{0}^{\infty}|\mathrm{f}(\mathrm{t})|^{\mathrm{q}} \Delta \mathrm{t}\right) .
$$

Proof. By Definition 6 we know that

$$
\mathrm{M}_{0}^{\alpha} \mathrm{f}(\mathrm{t})=\sup _{B \ni t} \frac{1}{|B|} \int_{0}^{\mathrm{t}} \mathrm{h}_{\alpha-2}(\mathrm{t}, \sigma(\tau)) \mathrm{f}(\tau) \Delta \tau
$$

Hence, by Hölder's inequality, we have

$$
\begin{gathered}
\left|\mathrm{M}_{0}^{\alpha} \mathrm{f}(\mathrm{t})\right| \leq \sup _{\mathrm{B} \ni \mathrm{t}} \frac{1}{|\mathrm{~B}|} \int_{\mathrm{a}}^{\mathrm{t}}\left|\mathrm{h}_{\alpha-2}(\mathrm{t}, \sigma(\tau)) \mathrm{f}(\tau)\right| \Delta \tau \\
\leq \sup _{\mathrm{B} \ni \mathrm{t}} \frac{1}{|\mathrm{~B}|}\left(\int_{0}^{\mathrm{t}}\left|\mathrm{h}_{\alpha-2}(\mathrm{t}, \sigma(\tau))\right|^{\mathrm{p}} \Delta \tau\right)^{\frac{1}{\mathrm{p}}}\left(\int_{0}^{\mathrm{t}}|\mathrm{f}(\tau)|^{\mathrm{q}} \Delta \tau\right)^{\frac{1}{\mathrm{q}}} \\
\leq \sup _{\mathrm{B} \ni \mathrm{t}} \frac{1}{|\mathrm{~B}|}\left(\int_{0}^{\mathrm{t}}\left|\mathrm{h}_{\alpha-2}(\mathrm{t}, \sigma(\tau))\right|^{\mathrm{p}} \Delta \tau\right)^{\frac{1}{\mathrm{p}}}\left(\int_{0}^{\infty}|\mathrm{f}(\tau)|{ }^{\mathrm{q}} \Delta \tau\right)^{\frac{1}{\mathrm{q}}}
\end{gathered}
$$


Herewith, the following result is obtained

$$
\left|\mathrm{M}_{0}^{\alpha} \mathrm{f}(\mathrm{t})\right|^{\mathrm{q}} \leq \sup _{\mathrm{B} \ni \mathrm{t}} \frac{1}{|\mathrm{~B}|}\left(\int_{0}^{\mathrm{t}}\left|\mathrm{h}_{\alpha-2}(\mathrm{t}, \sigma(\tau))\right|^{\mathrm{p}} \Delta \tau\right)^{\frac{\mathrm{q}}{\mathrm{p}}}\left(\int_{0}^{\infty}|\mathrm{f}(\tau)|^{\mathrm{q}} \Delta \tau\right) .
$$

If we take the integral of both sides of (13) through $\forall t \in[0, \infty)_{\mathbb{T}}$ we get

$$
\int_{0}^{\infty}\left|\mathrm{M}_{0}^{\alpha} \mathrm{f}(\mathrm{t})\right|^{\mathrm{q}} \Delta \mathrm{t} \leq\left(\int_{0}^{\infty} \sup _{\mathrm{B} \ni \mathrm{t}} \frac{1}{|\mathrm{~B}|}\left(\int_{0}^{\mathrm{t}}\left|\mathrm{h}_{\alpha-2}(\mathrm{t}, \sigma(\tau))\right|^{\mathrm{p}} \Delta \tau\right)^{\frac{\mathrm{q}}{\mathrm{p}}} \Delta \mathrm{t}\right)\left(\int_{0}^{\infty}|\mathrm{f}(\mathrm{t})|^{\mathrm{q}} \Delta \mathrm{t}\right) .
$$

Now, we present a few applications of our results.

\section{Applications}

Example 1. Let $f(t)=\left\{\begin{array}{c}t+1,1 \leq t<\frac{5}{2} \\ 2 t-1,3 \leq t<8\end{array}\right\}, g(t)=\left\{\begin{array}{c}t^{2}+1,1 \leq t<\frac{5}{2} \\ 3 t^{3}-2,3 \leq t<8\end{array}\right\} ; t \in \mathbb{T}=\mathbb{N}_{0}=\left\{n: n \in \mathbb{N}_{0}\right\}$ be two synchronous functions on $[0, \infty)_{\mathbb{T}}$. From Definition 3

$$
[\mathrm{f}(\mathrm{t})-\mathrm{f}(\mathrm{z})][\mathrm{g}(\mathrm{t})-\mathrm{g}(\mathrm{z})]=[\mathrm{t}+1-\mathrm{z}-1]\left[\mathrm{t}^{2}+1-\mathrm{z}^{2}-1\right]=[\mathrm{t}-\mathrm{z}]\left[\mathrm{t}^{2}-\mathrm{z}^{2}\right]=[\mathrm{t}-\mathrm{z}]^{2}[\mathrm{t}+\mathrm{z}] \geq 0
$$

and

$$
[\mathrm{f}(\mathrm{t})-\mathrm{f}(\mathrm{z})][\mathrm{g}(\mathrm{t})-\mathrm{g}(\mathrm{z})]=[2 \mathrm{t}-1-2 \mathrm{z}+1]\left[3 \mathrm{t}^{2}-2-3 \mathrm{z}^{2}+2\right]=6[\mathrm{t}-\mathrm{z}]^{2}[\mathrm{t}+\mathrm{z}] \geq 0 .
$$

Then, for $\forall t>a, a=1, \alpha=2$ we have

$$
M_{1}^{2}(f g)(t) \geq\left(h_{2}(t, 1)\right)^{-1}\left(M_{1}^{2} f\right)(t)\left(M_{1}^{2} g\right)(t) .
$$

Example 2. Let $f(t)=t+1, g(t)=t^{2}-1 ; t \in \mathbb{T}=\mathbb{N}_{0}=\left\{n: n \in \mathbb{N}_{0}\right\}$ be two synchronous functions on $[0, \infty)_{\mathbb{T}}$. Then $\forall t>a, a=1, \alpha=2, \beta=3$ we have

$$
\mathrm{h}_{2}(\mathrm{t}, 1) \mathrm{M}_{1}^{3}(\mathrm{fg})(\mathrm{t})+\mathrm{h}_{3}(\mathrm{t}, 1) \mathrm{M}_{1}^{2}(\mathrm{fg})(\mathrm{t}) \geq\left(\mathrm{M}_{1}^{2} \mathrm{f}\right)(\mathrm{t})\left(\mathrm{M}_{1}^{3} \mathrm{~g}\right)(\mathrm{t})+\left(\mathrm{M}_{1}^{3} \mathrm{f}\right)(\mathrm{t})\left(\mathrm{M}_{1}^{2} \mathrm{~g}\right)(\mathrm{t}) .
$$

Example 3. Let $\left(f_{i}\right)_{i=1, \ldots, 9} f_{i}(t)=\left(2 t^{i}+12\right) ; t \in \mathbb{T}=\mathbb{N}_{0}=\left\{n: n \in \mathbb{N}_{0}\right\}$ be a synchronous function on $[0, \infty)_{\mathbb{T}}$. Then, for $\forall t>a, a=2, \alpha=3$ we have

$$
\mathrm{M}_{2}^{3}\left(\prod_{\mathrm{i}=1}^{9} \mathrm{f}_{\mathrm{i}}\right)(\mathrm{t}) \geq\left(\mathrm{h}_{3}(\mathrm{t}, 2)\right)^{-8} \prod_{\mathrm{i}=1}^{9} \mathrm{M}_{2}^{3} \mathrm{f}_{\mathrm{i}}(\mathrm{t}) .
$$

Remark 1. Let $p: \Phi \rightarrow[1, \infty)$ be a measurable function for $\Phi \subset \mathbb{R}$. Using Definition 2 , we can easily see that the following norm inequalities are provided in the variable exponential Lebesgue space.

(I) $\quad\left\|\mathrm{M}_{\mathrm{a}}^{\alpha}(\mathrm{fg})(\mathrm{t})\right\|_{\mathrm{L}^{\mathrm{p}(\mathrm{x})}\left([0, \infty)_{\mathbb{T}}\right)} \geq\left\|\left(\mathrm{h}_{\alpha}(\mathrm{t}, \mathrm{a})\right)^{-1}\left(\mathrm{M}_{\mathrm{a}}^{\alpha} \mathrm{f}\right)(\mathrm{t})\left(\mathrm{M}_{\mathrm{a}}^{\alpha} \mathrm{g}\right)(\mathrm{t})\right\|_{\mathrm{L}^{\mathrm{p}(\mathrm{x})}\left([0, \infty)_{\mathbb{T}}\right)}$

(II) $\quad\left\|h_{\alpha}(t, a) M_{a}^{\beta}(f g)(t)+h_{\beta}(t, a) M_{a}^{\alpha}(f g)(t)\right\|_{L^{p(x)}\left([0, \infty)_{\mathbb{T}}\right)}$

$$
\geq\left\|\left(M_{a}^{\alpha} f\right)(t)\left(M_{a}^{\beta} g\right)(t)+\left(M_{a}^{\beta} f\right)(t)\left(M_{a}^{\alpha} g\right)(t)\right\|_{L^{p}(x)}\left([0, \infty)_{\mathbb{T}}\right)
$$

(III) $\left\|M_{a}^{\alpha}\left(\prod_{i=1}^{n} f_{i}\right)(t)\right\|_{L^{p(x)}\left([0, \infty)_{\mathbb{T}}\right)} \geq\left\|\left(h_{\alpha}(t, a)\right)^{1-n} \prod_{i=1}^{n} M_{a}^{\alpha} f_{i}(t)\right\|_{L^{p(x)}\left([0, \infty)_{\mathbb{T}}\right)}$ 


\section{Discussion and Conclusions}

Recently, the concept of inequalities and dynamic equations in time scales has gained an important place in the scientific literature. Mathematicians have emphasized many aspects of integral inequalities and integral equation, for example, transformations, inverse conversions, extensions, etc. However, these studies did not work on time scales. Moreover, the contribution of these studies to science has been weak. In particular, apart from the science of mathematics, we see very little the effect of the concept of time scales in different science fields. This study motivated us to find solutions to problems in these areas. In this paper, we examined fractional maximal integral inequalities on time scales. Furthermore, we demonstrated that different results can be obtained. These results can be examined in two or more dimensions. Moreover, they can be extended to nabla and diamond alpha derivatives.

Funding: This research received no external funding.

Acknowledgments: The author is grateful to the editor and the referees for their careful reading of the manuscript and their valuable comments.

Conflicts of Interest: The author declares no conflict of interest.

\section{References}

1. Hilger, S. Analysis on measure chains-a unified approach to continuous and discrete calculus. Results Math. 1990, 18, 18-56. [CrossRef]

2. Tassaddiq, A.; Rahman, G.; Nisar, K.S.; Samraiz, M. Certain fractional conformable inequalitiesfor the weighted and the extended Chebyshev functionals. Adv. Differ. Equ. 2020, 2020, 1-9. [CrossRef]

3. Singh, J.; Kumar, D.; Hammouch, Z.; Atangana, A. A fractional epidemiological model for computer viruses pertaining to a new fractional derivative. Appl. Math. Comput. 2018, 316, 504-515. [CrossRef]

4. Dananea, J.; Allalia, K.; Hammouch, Z. Mathematical analysis of a fractional differential model of HBV infection with antibody immune response. Chaos Solut. Fractals 2019, 136, 109787. [CrossRef]

5. Ghanbari, B.; Günerhan, H.; Srivastava, H.M. An application of the Atangana-Baleanu fractional derivative in mathematical biology: A three-species predator-prey model. Chaos Solut. Fractals 2020, 138, 109910. [CrossRef]

6. Bohner, M.; Peterson, A. Dynamic Equations on Time Scales, An İntroduction with Applications; Birkhauser: Boston, MA, USA, 2001. [CrossRef]

7. Akin-Bohner, E.; Bohner, M.; Akin, F. Pachpatte inequalities on time scales. J. Inequal. Pure Appl. Math. 2005, $6,1-23$.

8. Li, W.N. Nonlinear Integral Inequalities in Two Independent Variables on Time Scales. Adv. Differ. Equ. 2011, 283926. [CrossRef]

9. Kac, V.; Cheung, P. Quantum Calculus; Universitext Springer: New York, NY, USA, 2002.

10. Chen, Q.; Yang, B. On a new reverse Hardy-Littlewood's type inequality. Appl. Math. Sci. 2012, 6, 6553-6561.

11. Bohner, M.; Heim, J.; Liu, A. Qualitative analysis of Solow model on time scales. J. Concrete Appl. Math. 2015, 13, 183-197.

12. Agarwal, R.P.; Bohner, M.; Peterson, A. Inequalities on time scales: A survey. Math. Inequal. Appl. 2001, 4, 535-555. [CrossRef]

13. Belarbi, S.; Dahmani, Z. On some new fractional integral inequalities. J. Inequal. Pure Appl. Math. 2009, 10, $1-12$.

14. Bohner, M.; Guseinov, G.S. Multiple Lebesgue integration on time scales. Adv. Differ. Equ. 2006, 026391. [CrossRef]

15. Agarwal, R.P.; Otero-Espinar, V.; Perera, K.; Vivero, D.R. Basic properties of Sobolev's spaces on time scales. Adv. Differ. Equ. 2006, 038121. [CrossRef]

16. Chen, G.; Wei, C. A functional generalization of diamond- $\alpha$ integral Dresher's inequality on time scales. Adv. Differ. Equ. 2014, 2014, 324. [CrossRef]

17. Rahman, G.; Nisar, K.S.; Abdeljawad, T. Certain Hadamard Proportional Fractional Integral Inequalities. Mathematics 2020, 8, 504. [CrossRef] 
18. Rahman, G.; Nisar, K.S.; Abdeljawad, T. Tempered Fractional Integral Inequalities for Convex Functions. Mathematics 2020, 8, 500. [CrossRef]

19. Ucar, S.; Ucar, E.; Ozdemir, N.; Hammouch, Z. Mathematical analysis and numerical simulation for a smoking model with Atangana-Baleanu derivative. Chaos Solut. Fractals 2019, 118, 300-306. [CrossRef]

20. Chebyshev, P.L. Sur les expressions approximatives des integrales definies par les autres prises entre les memes limites. Proc. Math. Soc. Charkov. 1882, 2, 93-98.

21. Akin, L. Compactness of Fractional Maximal Operator in Weighted and Variable Exponent Spaces. Erzincan Univ. J. Sci. Technol. 2019, 12, 185-190. [CrossRef]

22. Uçar, D.; Hatipoğlu, V.F.; Akincali, A. Fractional Integral Inequalıties On Tıme Scales. Open J. Math. Sci. 2018, 2,361-370. [CrossRef]

23. Agarwal, R.P.; O’Regan, D.; Saker, S.H. Dynamic Inequalities on Time Scales; Springer: Cham, Switzerland, 2014.

24. Bohner, M.; Agarwal, R.P. Basic calculus on time scales and some of its applications. Result. Math. 1999, 35, 3-22. [CrossRef]

25. Anastassiou, G.A. Principles of delta fractional calculus on time scales and inequalities. Math. Comput. Model. 2010, 52, 556-566. [CrossRef]

(C) 2020 by the author. Licensee MDPI, Basel, Switzerland. This article is an open access article distributed under the terms and conditions of the Creative Commons Attribution (CC BY) license (http://creativecommons.org/licenses/by/4.0/). 